\title{
Freqüência dos tipos de desvios oculares no ambulatório de motilidade ocular extrínseca do Hospital do Servidor Público Estadual de São Paulo
}

\author{
Frequency of ocular deviations at the strabismus sector of the Hospital do \\ Servidor Público Estadual de São Paulo
}

\author{
Marcelo Jarczun Kac ${ }^{1}$ \\ Moacyr Borges de Freitas Júnior ${ }^{2}$ \\ Sansão Isaac Kac ${ }^{3}$ \\ Eric Pinheiro de Andrade
}

Trabalho realizado no Departamento de Oftalmologia do Hospital do Servidor Público Estadual "Francisco Morato de Oliveira - HSPE-FMO" - São Paulo (SP) Brasil.

Residente do $3^{\circ}$ ano do Hospital do Servidor Público Estadual "Francisco Morato de Oliveira - HSPE-FMO" - São Paulo (SP) - Brasil.

${ }^{2}$ Residente do $1^{\circ}$ ano do Hospital do Servidor Público Estadual "Francisco Morato de Oliveira - HSPE-FMO" - São Paulo (SP) - Brasil.

${ }^{3}$ Chefe do Setor de Oftalmopediatria do Hospital dos Servidores do Estado - HSE - Rio de Janeiro (RJ) - Brasil.

${ }^{4}$ Chefe do Ambulatório de Neuroftalmologia do Hospital do Servidor Público Estadual "Francisco Morato de Oliveira - HSPE-FMO" - São Paulo (SP) - Brasil.

Endereço para correspondência: Marcelo Jarczun Kac. Av. Nossa Senhora de Copacabana, 680/1203 - Rio de Janeiro (RJ) CEP 22050-000

E-mail: marcelojkac@gmail.com

Recebido para publicação em 24.11.2006

Última versão recebida em 04.07.2007

Aprovação em 06.08.2007

Nota Editorial: Depois de concluída a análise do artigo sob sigilo editorial e com a anuência da Dra. Maria de Lourdes Motta Moreira Villas Boas sobre a divulgação de seu nome como revisora, agradecemos sua participação neste processo.

\begin{tabular}{l} 
RESUMO \\
\hline Objetivo: Identificar os tipos de desvios oculares e suas freqüências nos \\
pacientes encaminhados ao ambulatório de motilidade ocular extrínseca \\
do Hospital do Servidor Público Estadual de São Paulo. Métodos: Foram \\
revisados 935 prontuários do ambulatório de motilidade ocular extrínseca \\
do ano de 2005 . Os pacientes foram avaliados quanto à idade, sexo tipo \\
de desvio apresentado na posição primária do olhar, sendo submetidos \\
esses dados à avaliação estatística. Resultados: A esotropia foi o desvio \\
com maior prevalência na população estudada (44,52\%). Neste grupo \\
houve predomínio de indivíduos do sexo masculino ( $\mathrm{p}=0,001$ ). Este \\
desvio foi mais freqüente na faixa etária de $0-2$ anos (p=0,009). Já a \\
exotropia (12,25\%) mostrou-se mais freqüente entre as mulheres (p=0,046), \\
sendo predominante na faixa etária superior aos 47 anos de idade \\
(p=0,001) Conclusão: Observou-se maior número de esodesvios em \\
relação aos exodesvios. As esotropias foram mais freqüentes no sexo \\
masculino em faixas etárias mais jovens. Já as exotropias se apresentaram \\
em maior número no sexo feminino em faixas etárias mais elevadas. O \\
maior número de exoforias foi encontrado em pacientes com idades entre \\
16 e 20 anos.
\end{tabular}

Descritores: Estrabismo/classificação; Estrabismo/epidemiologia; Estrabismo/diagnóstico; Prevalência; Coleta de dados

\section{INTRODUÇ̃̃̃O}

Estrabismo é o desalinhamento ocular. Pode ser constante (tropia) ou latente, manifestando-se apenas quando há quebra da fusão (foria).

Ocorrendo precocemente, pode acarretar na alteração da adaptação sensorial, resultando em supressão, correspondência retiniana anômala, e/ ou ambliopia. Quando surge tardiamente, mais precisamente após a maturação binocular, gera confusão de imagem ou diplopia, podendo persistir indefinidamente ou até a correção motora do desvio ${ }^{(1)}$. Alterações posturais com posição anômala da cabeça ${ }^{(2)}$ podem ocorrer na tentativa compensatória de distúrbios da visão binocular ocasionados pelo estrabismo.

Nos adultos pode ocorrer secundariamente a doenças neurológicas, doenças vasculares como no diabetes mellitus e na hipertensão arterial, doenças da tireóide ${ }^{(3)}$, tumores cerebrais e traumas cranianos.

Quando existe uma redução da acuidade visual (secundária a catarata, ametropias, anisometropias ou outras lesões), há o surgimento de um estrabismo devido à formação defeituosa da imagem, não havendo estímulo para a fusão. 
Os desvios oculares podem se manifestar no plano horizontal (esodesvios e exodesvios) e/ou ciclovertical (hipertropias, torsões e desvios verticais dissociados). Eles podem ser constantes ou intermitentes.

Os principais objetivos do tratamento são: a) preservar e garantir desenvolvimento adequado da visão; b) recuperar a visão binocular; e c) estabelecer o paralelismo ocular. Dependendo da causa, o tratamento pode ser clínico, óptico, cirúrgico ou misto.

O objetivo do presente estudo consiste na identificação da freqüência dos desvios oculares em pacientes encaminhados ao ambulatório de motilidade ocular extrínseca do Hospital do Servidor Público Estadual de São Paulo, no ano de 2005, correlacionando sexo e faixa etária, além de comparar com os dados disponíveis na literatura.

\section{MÉTODOS}

Foi realizado um estudo retrospectivo de todos os pacientes atendidos no Ambulatório de Motilidade Ocular Extrínseca da Clínica de Oftalmologia do Hospital do Servidor Público Estadual de São Paulo no ano de 2005.

Os pacientes foram alocados conforme sexo, idade (no dia da consulta), presença ou ausência de desvio ocular, além do tipo de desvio na posição primária do olhar presente no momento da consulta.

Ao alocarmos conforme a idade, foi realizada divisão etária baseando-se na literatura ${ }^{(4)}$ :

Grupo I - 0 a 2 anos

Grupo II - 3 a 5 anos

Grupo III - 6 a 10 anos

Grupo IV - 11 a 15 anos

Grupo V - 16 a 20 anos

Grupo VI - 21 a 46 anos

Grupo VII - 47 ou mais anos

Com relação aos desvios oculares na posição primária do olhar, os pacientes foram alocados nos seguintes grupos:

Grupo 1 - Esoforia

Grupo 2 - Esotropia intermitente

Grupo 3 - Esotropia

Grupo 4 - Exoforia

Grupo 5 - Exotropia intermitente

Grupo 6 - Exotropia

Grupo 7 - Desvio vertical dissociado (DVD)

Grupo 8 - Hipertropia intermitente

Grupo 9 - Hipertropia

Grupo 10 - Ausência de desvio.

Os resultados foram submetidos à análise estatística pelos testes de Fisher e Qui-quadrado, utilizando como nível de significância valores inferiores a 0,05 .

Esta pesquisa seguiu os princípios básicos contidos na Declaração de Helsinque (1964) e foi aprovada pela Comissão de Ética em Pesquisa do Instituto de Assistência Médica ao Servidor Público Estadual de São Paulo - Hospital do Servidor Público Estadual (CEP nº 064/06).

\section{RESULTADOS}

Foram avaliados 935 prontuários atendidos no período citado. Quinhentos e nove eram do sexo feminino $(54,44 \%)$ e 426 do sexo masculino (45,56\%) (Quadro 1). Duzentos e quarenta e um pacientes $(25,78 \%)$ não apresentavam desvio ocular na posição primária do olhar, enquanto que os 694 restantes $(74,22 \%)$ foram classificados quanto ao tipo de desvio apresentado: 35 (5,04\%) esoforia, 87 (12,54\%) esotropia intermitente, $309(44,52 \%)$ esotropia, $65(9,37 \%)$ exoforia, 54 $(7,78 \%)$ exotropia intermitente, $85(12,25 \%)$ exotropia, 5 $(0,72 \%)$ apresentavam DVD, $2(0,29 \%)$ hipertropia intermitente e $52(7,49 \%)$ hipertropia (Quadro 2).

Quanto à distribuição pelo sexo, 366 pacientes $(52,74 \%)$ eram do sexo feminino enquanto $328(47,26 \%)$ eram do sexo masculino.

Alocando os pacientes conforme a faixa etária, $10(1,44 \%)$ pertenciam ao grupo I, $62(8,93 \%)$ ao grupo II, $206(29,68 \%)$ ao grupo III, $146(21,04 \%)$ ao grupo IV, $76(10,95 \%)$ ao grupo V, 96 $(13,83 \%)$ ao grupo VI e $98(14,12 \%)$ ao grupo VII (Quadro 3).

\begin{tabular}{|lcc|}
\hline \multicolumn{3}{|c|}{ Quadro 1. Distribuição dos pacientes segundo o sexo } \\
Sexo & Número de pacientes & Porcentagem \\
Feminino & 509 & $54,44 \%$ \\
Masculino & 426 & $45,56 \%$ \\
Total & 935 & $100,00 \%$ \\
\hline
\end{tabular}

Quadro 2. Distribuição dos pacientes segundo o tipo de desvio apresentado

\begin{tabular}{lrr} 
Desvio & Número de pacientes & Porcentagem \\
Esoforia & 35 & $5,04 \%$ \\
Esotropia intermitente & 87 & $12,54 \%$ \\
Esotropia & 309 & $44,52 \%$ \\
Exoforia & 65 & $9,37 \%$ \\
Exotropia intermitente & 54 & $7,78 \%$ \\
Exotropia & 85 & $12,25 \%$ \\
DVD & 5 & $0,72 \%$ \\
Hipertropia intermitente & 2 & $0,29 \%$ \\
Hipertropia & 52 & $7,49 \%$ \\
Total & 694 & $100,00 \%$ \\
\hline
\end{tabular}

\begin{tabular}{|ccc|}
\hline \multicolumn{3}{|c|}{ Quadro 3. Distribuição dos pacientes segundo a faixa etária } \\
Faixa etária (anos) & Número de pacientes & Porcentagem \\
$0-2$ & 10 & $1,44 \%$ \\
$3-5$ & 62 & $8,94 \%$ \\
$6-10$ & 206 & $29,68 \%$ \\
$11-15$ & 146 & $21,04 \%$ \\
$16-20$ & 76 & $10,95 \%$ \\
$21-46$ & 96 & $13,83 \%$ \\
47 ou mais & 98 & $14,12 \%$ \\
Total & 694 & $100,00 \%$ \\
\hline
\end{tabular}


Comparando o tipo de desvio relacionado ao sexo foi observada uma diferença estatisticamente significativa em dois grupos: a esotropia foi mais freqüente no sexo masculino $(p=0,001)$ enquanto que na exotropia, o desvio foi mais freqüente no sexo feminino $(p=0,046)$. Nos outros tipos de desvios não foram observadas diferenças significativas quanto à freqüência em ambos os sexos.

Correlacionando a faixa etária com o tipo de desvio, a esotropia intermitente foi mais prevalente no grupo II $(\mathrm{p}=0,001)$. Esta relação se mantém ao analisar os pacientes pelo sexo. A esotropia se mostra mais freqüente no grupo I $(p=0,009)$, relação também verificada entre pacientes do sexo masculino nesta faixa etária $(\mathrm{p}=0,002)$. Contudo, não possui relevância estatística no grupo feminino $(\mathrm{p}=0,477)$.

Já a exoforia se mostrou predominante no grupo $\mathrm{V}$ $(p=0,004)$. No sexo feminino esta relação se mantém $(p=0,026)$; porém não ocorre o mesmo no sexo masculino $(\mathrm{p}=0,184)$. Nas exotropias observamos uma maior freqüência no grupo VII $(\mathrm{p}=0,001)$, mantendo esta relação ao analisar tanto o sexo feminino $(p=0,001)$ quanto o masculino $(p<0,001)$, isoladamente.

Com relação à hipertropia, verificamos uma maior freqüência no grupo VII ( $\mathrm{p}=0,001)$. Analisando somente pacientes do sexo masculino, esta relação é mantida $(\mathrm{p}<0,001)$. Entretanto, ao analisar o sexo feminino, verificamos uma maior presença de hipertropia no grupo I seguido do grupo VII $(\mathrm{p}<0,001)$.

Não foi observada relação estatisticamente significativa entre os desvios e suas ocorrências por faixas etárias nas esoforias, exotropias intermitentes, DVD, e hipertropias intermitentes.

\section{DISCUSSÃO}

A prevalência de desvios convergentes quando comparada com desvios divergentes é maior em europeus e seus descendentes ${ }^{(5-6)}$, contudo, na Ásia, as exotropias são mais prevalentes $^{(7)}$. No Brasil não encontramos estudos correlacionando o tipo de desvio com etnia, visto a grande variabilidade de raças encontradas. $\mathrm{O}$ presente estudo verificou que o número de desvios convergentes concentrou aproximadamente $62 \%$ do total de desvios encontrados, concordando com a grande maioria dos estudos europeus e americanos ${ }^{(5,8-11)}$, onde o número de desvios convergentes se sobrepõem aos desvios divergentes.

Quando foram comparados os desvios aos sexos, foi observada maior prevalência de esotropias no sexo masculino. Outros estudos não encontraram diferenças quanto ao sexo na presença de esotropias ${ }^{(7)}$. Porém é importante ressaltar que esta característica não foi estudada em vários trabalhos ${ }^{(4-6,12-13)}$.

Já nas exotropias, encontramos um maior número de casos no sexo feminino. Nusz e et al. verificaram uma maior ocorrência de exotropias intermitentes no sexo feminino ${ }^{(14)}$, contudo, não conseguiu estabelecer uma explicação para tal fato. Outros estudos, já não verificaram a presença de diferenças quanto ao sexo nas exotropias ao analisar pacientes com idade inferior a $19 \operatorname{anos}^{(15)}$.

Em relação aos desvios divergentes, observamos um maior número de casos de exotropias, seguido por exoforias e exotropias intermitentes, respectivamente. Outros autores observaram que a exotropia intermitente foi o desvio mais freqüente $^{(15-16)}$. Em estudo realizado na cidade de Natal - RN - Brasil, encontrou-se um maior número de exotropias do que exotropias intermitentes ${ }^{(4)}$.

Ao comparar os tipos de desvios relacionados às diferentes faixas etárias, pudemos verificar um grande número de casos de esotropias e esotropias intermitentes nos pacientes dos grupos I e II. Isso provavelmente se deve ao grande número de casos de esotropias congênitas, esotropias comitantes adquiridas e esotropias acomodativas observadas nessas faixas etárias ${ }^{(5)}$.

As exotropias se mostraram mais prevalentes no grupo VII. $\mathrm{Na}$ literatura, encontramos um trabalho envolvendo pacientes acima de 60 anos, onde os desvios horizontais foram divididos de modo similar entre esotropias e exotropias ${ }^{(12)}$. Já as exoforias se apresentam mais prevalentes no grupo V. A utilização excessiva de microcomputadores, estudos e leitura podem estar contribuindo para termos este tipo de descompensação nesta faixa etária.

As hipertropias foram verificadas em maior número no grupo VII.

\section{CONCLUSÃO}

O estudo demonstrou que a presença de desvios convergentes é superior a de desvios divergentes na população analisada. Além disso, os desvios convergentes se concentram nas faixas etárias mais jovens (entre 0-5 anos). Também foi constatada uma maior ocorrência de esotropias no sexo masculino. Já as exotropias se apresentaram mais freqüentemente na população acima dos 47 anos de idade, e predominantemente no sexo feminino. As exoforias apresentaram um maior número de casos em pacientes entre 16-20 anos.

Este trabalho ajuda a traçar um quadro da prevalência de desvios oculares na nossa população e suas peculiaridades. Mais estudos são necessários para determinar com maior precisão as características dos pacientes portadores de estrabismo na população brasileira.

\section{ABSTRACT}

Purpose: To evaluate the frequency of different types of ocular deviations in patients referred to the strabismus sector at the Hospital do Servidor Público Estadual de São Paulo - Brazil. Methods: A retrospective study of 935 records of patients examined at the strabismus sector in 2005 was conducted to evaluate sex, age and types of misalignments in the primary position. Statistical analysis was applied to these data. Re- 
sults: Esotropia was the most prevalent misalignment in this population $(44.52 \%)$. There were more males in this group $(\mathrm{p}=0.001)$ with a predominance of the age group $0-2$ years $(p=0.009)$. Exotropia $(12.25 \%)$ was more frequent in females $(p=0.046)$ in the age group 46 years and over $(p=0.001)$. Conclusion: Esodeviations were more frequent than exodeviations. Esotropia was more prevalent in males early in life, while exotropia was more frequent in older females. The larger number of exophorias was found in the age group 16-20 years.

Keywords: Strabismus/classification; Strabismus/epidemiology; Strabismus/diagnosis; Prevalence; Data collection

\section{REFERÊNCIAS}

1. Bicas HEA. Visão binocular e estrabismo. Arq Bras Oftalmol. 1987;50(1):36-41.

2. Bicas HEA, Caldeira JAF, Campos WR, Carvalho KMM, Cukierman S, Cunha RL, et al. Rotina de exames do estrabismo: caderno 3. Arq Bras Oftalmol. 1987;50(1):13-35.

3. Volpe NJ, Sbarbaro JA, Gendron Livingston K, Galetta SL, Liu GT, Balcer LJ. Occult thyroid eye disease in patients with unexplained ocular misalignment identified by standardized orbital echography. Am J Ophthalmol. 2006; 142(1):75-81.
4. Garcia CAA, Sousa AB, Mendonça MBM, Andrade LL, Oréfice F. Prevalence of strabismus among students in Natal/RN - Brazil. Arq Bras Oftalmol 2004;67(5):791-4.

5. Prieto-Díaz J, Souza-Dias C. Estrabismo. 4ำ ed. São Paulo: Livraria Santos; 2002. p.149-50.

6. Friedman Z, Neumann E, Hyams SW, Peleg B. Ophthalmic screening of 38,000 children, age 1 to $2 \frac{1}{2}$ years, in child welfare clinics. J Pediatr Ophthalmol Strabismus. 1980;17(4):261-7.

7. Yu CB, Fan DS, Wong VW, Wong CY, Lam DS. Changing patterns of strabismus: a decade of experience in Hong Kong. Br J Ophthalmol. 2002; 86(8):854-6. Comment in: Br J Ophthalmol. 2002;86(8):835-6.

8. Preslan MW, Novak A. Baltimore Vision Screening Project. Ophthalmology. 1996;103(1):105-9.

9. Preslan MW, Novak A. Baltimore Vision Screening Project. Phase 2. Ophthalmology. 1998;105(1):150-3.

10. Kvarnström G, Jakobsson P, Lennerstrand G. Visual screening of Swedish children: an ophthalmological evaluation. Acta Ophthalmol Scand. 2001; 79(3):240-4.

11. Sá LCF. Estrabismo nos anos 90. Arq Bras Oftalmol. 1998;61(6):720-4

12. Magramm I, Schlossman A. Strabismus in patients over the age of 60 years. J Pediatr Ophthalmol Strabismus. 1991;28(1):28-31.

13. Carney CV, Lysons DA, Tapley JV. Is the incidence of constant esotropia in childhood reducing? Eye. 1995;9(Pt $6 \mathrm{Su}): 40-1$

14. Nusz KJ, Mohney BG, Diehl NN. Female predominance in intermittent exotropia. Am J Ophthalmol. 2005;140(3):546-7.

15. Govindan M, Mohney BG, Diehl NN, Burke JP. Incidence and types of childhood exotropia: a population-based study. Ophthalmology. 2005;112 (1):104-8.

16. Mohney BG, Huffaker RK. Common forms of childhood exotropia. Ophthalmology. 2003;110(11):2093-6. 